\title{
Synthetic Biology and Sustainable Energy
}

\author{
Y.J.CHEN \\ Institute of Scientific and Technical Information of China, Beijing, China
}

\begin{abstract}
Synthetic biology can be defined as a combinatorial approach where the basic elements, governing the operational functionality of a living cell, are placed together to generate life forms with novel characteristics and properties. One of the most exciting aspects of synthetic biology is the diversity of applications it may enable. New applications may be found in energy, medicine, environment and materials. In this paper, we first focus on the definition of synthetic biology as well as the potential applications and key challenges, then the role of synthetic biology in producing biofuels are discussed. Finally, several key points to achieve sustainable development are summarized.
\end{abstract}

KEYWORD: synthetic biology; biofuel; microbiology; sustainable energy

\section{INTRODUCTION}

Synthetic biology can be considered as extreme genetic engineering as it is an amalgamation of the principles of engineering and biology that provide a new paradigm for generation of novel applications in diverse areas such as therapeutics, bioremediation, waste resource management and biofuel production [1-5]. It is a multidisciplinary science in a true sense as it derives knowledge from various disciplines (such as genetics, nanotechnology, chemical engineering, robotics, systems biology and computational biology), in order to manipulate living cells to obtain desired functions.

Over the past decade, synthetic biology has emerged as an engineering discipline for biological systems. Compared with other substrates, biology possess a unique set of engineering challenges resulting from an incomplete understanding of natural biological systems and tools for manipulating them. It is speculated that with further technological advancements and understanding of the biological phenomenon, greater avenues for synthetic biology applications will emerge which would allow incorporation of the information generated through various projects based on genomics, proteomics, metabolomics and phenomics [6].

Synthetic biology aims at enablement of creation of living organisms with user defined genetic circuitry and novel characteristics by modulating, regulating and redesigning the gene expression profile of the host organism, enabling its exploitation for various applications [7]. As a stepping stone towards this direction, micro-organisms (such as Escherichia coli) with minimal genome have been created [8]. Such microbes can be further transformed with genes conferring them novel functions.

On the other hand, microbiology, the multidisciplinary science of microorganisms, has a significant impact on the world. With its roots in the 19 th century, it matured into a scientific discipline concerned with the structure, function, and classification of these organisms and with ways of controlling and using their activities. It continued to evolve into the major subdivisions of microbiology, which include medical, industrial, agricultural, food and dairy. Microbiology is used to treat major pandemics and frequent infections. It also has an impact on the food people consume [9], and it has been shown that the recently identified natural antitumor compounds from various natural origins including fungi and endophytic fungi [10].

The present study focuses on applications of synthetic biology in producing biofuel and achieving sustainable development.

\section{SYNTHETIC BIOLOGY: POTENTIAL APPLICATIONS AND KEY CHALLENGES}

Synthetic biology is an emerging field combining biology with engineering but lacks a coherent and agreed definition. Synthetic biology is perhaps best 
understood in relation to its aims, which are described by the OECD as being "to design and build new biological parts and systems or to modify existing ones to carry out novel tasks". [11] In other words, synthetic biology is

-the design and construction of new biological parts, devices and systems, and

-the redesign of existing, natural biological systems for useful purposes.

On the other hand, Murray did place the technologies comprising synthetic biology into four categories [12]:

-Advanced genetic engineering. Engineering bacteria to produce a precursor of artemisinin, a front-line malaria drug, epitomizes this category.

-DNA-based device construction. The BioBricks project applies the principles of electronics engineering to biology, aiming to one day build functional nano devices de novo from genes and proteins.

- Creating a minimal cell. Venter's achieve-ment of synthesizing a complete, functional genome for Mycoplasma mycoides is the leading indicator.

- Creating a protocell. According to Murray the goal is to create a new form of life. A genuinely new living entity, not based on the biology we've known thus far. This goal remains distant, but may lead to radical advances such as silicon-based life.

For the wider community the importance of synthetic biology lies in its social and commercial potential. One estimate suggests that the global market for synthetic biology could reach US\$2.4 billion by 2013, with applications ranging from medicine to agriculture [13]. Possible uses of synthetic biology include the following areas:

-Energy Custom-built microbes for generating hydrogen and other fuels, or for performing artificial photosynthesis. And the development of new pathways for producing fuel.

-Medicine and Health The manufacture of drugs, vaccines and diagnostic agents, and the creation of new tissue. Also including enhanced drug production and delivery.

-Environment The detection of pollutants, and their breakdown or removal from the environment; and including engineered dispersants and environmentally friendly materials.

- Chemical Industry The production of fine or bulk chemicals, including proteins to provide an alternative to natural fibres or existing synthetic fibres.

-Agriculture and Food Including engineered or optimized crops, and novel food additives.

Which of these applications will be first to make an impact in the marketplace is a matter of speculation, although many commentators foresee biofuel products as a likely frontrunner.

However, challenges loom at every step in the process, from the characterization of parts to the design and construction of systems. It has been pointed out that there are five key challenges [14]:

-Many of the parts are undefined A biological part can be anything from a DNA sequence. The problem is that many parts have not been characterized well. They haven't always been tested to show what they do, and even when they have, their performance can change with different cell types or under different laboratory conditions.

- The circuitry is unpredictable Even if the function of each part is known, the parts may not work as expected when put together. Synthetic biologists are often caught in a laborious process of trial-and-error, unlike the more predictable design procedures found in other modern engineering disciplines.

- The complexity is unwieldy As circuits get larger, the process of constructing and testing them becomes more daunting. For example, the researchers had to test many part variants before they found a configuration.

- Many parts are incompatible Once constructed and placed into cells, synthetic genetic circuits can have unintended effects on their host.

-Variability crashes the system Synthetic biologists must also ensure that circuits function reliably. Molecular activities inside cells are prone to random fluctuations, or noise. Variation in growth conditions can also affect behavior.

\section{SYNTHETIC BIOLOGY FOR BIOFUELS}

As the fossil fuel economy grows increasingly unsustainable, it becomes more and more important that humanity develops alternative energy solutions. Climate change is occurring at an alarming pace, disrupting the biosphere, facilitating international conflicts over finite resources, and destabilizing the global economy. Synthetic biology provides scientists with a path towards rapid development of renewable fuels via biological systems and this a feasible route for realizing global energy sustainability[15]. For instance, the most widely used biofuel is ethanol produced from corn or sugar cane [16]; however, the heavy agricultural burden combined with the suboptimal fuel properties of ethanol make this approach to biofuels problematic and limited. Microorganisms engineered with optimized biosynthetic pathways to efficiently convert biomass or non-edible vegetable oils into biofuels are an alternative and promising source of renewable energy [17]. These strategies will succeed only if their production costs can be made to compete with, or even outcompete, current fuel production costs.

For biofuel production, certain microorganisms have evolved to be proficient in converting lignocellulosic material to ethanol, biobutanol and 
other biofuels. These native isolates possess unique catabolic activity, heightened tolerances for toxic materials and a host of enzymes designed to break down the lignocellulosic components. Unfortunately, these highly desired properties exist in pathways that are tightly regulated according to the host's evolved needs and therefore may not be suitable in their native state for production scale. A longstanding challenge in metabolic and genetic engineering is determining whether to improve the isolate host's production capacity or whether to transplant the desired genes or pathways into an industrial model host, such as E. coli or S. cerevisiae [18].

Synthetic biology might accelerate the development of second-generation biofuels that can be prepared from agricultural waste and plant residues, so avoiding competition with crops grown for food. Synthetic biology involves engineering microbes to produce specifically desired fuels, especially hydrocarbon fuels that are "drop-in" replacements for petroleum diesel and gasoline. The work that has been done thus far has been targeting the development of microbes that "eat" sugar molecules and excrete diesel-like fuel [19].

On the other hand, synthetic biology will also be key in rewiring existing regulation. The design of new regulatory pathways from synthetic genetic elements will be important in sensing the extracellular or intracellular environment and producing a pro-grammed cellular response. For biofuel production from lignocellulosic biomass, this is of great importance as the efficient uptake of a mixture of hexose and pentose sugars simultaneously is desirable. Synthetic biology can provide tools to construct new circuits devoid of unwanted regulation from the bottom up [20] to sense the extracellular environment and produce the necessary response to digest the sugars. Ultimately, the bottom-up construction of biological circuits may extend beyond individual pathways toward all of metabolism, as the synthesis of entirely synthetic, replicable, and functional genomes has recently been accomplished [21]. In the future, this will allow synthetic biologists to build heterologous pathways into organisms devoid of unwanted pathways or properties.

\section{KEY POINTS FOR SUSTAINABLE DEVELOPMENT}

According to definition of sustainable energy, damage of environment is another major concern for sustainable energy development. Environmental efficiency is closely linked to working of sustainable and renewable energy development concerns. The term eco-efficiency was coined by the World Business Council for Sustainable Development (WBCSD). It is based on the concept of creating more goods and services while using fewer resources and creating lesser waste and pollution. WBCSD has pointed out seven major elements in considering eco-efficiency of developing environ-mental friendly products or processes for reducing environmental impacts [22].

-Reduce the material intensity of its goods and services.

- Reduce the energy intensity of its goods and services.

-Reduce the dispersion of any toxic materials.

-Enhance the recyclability of its materials.

-Maximize the sustainable use of renewable resources.

-Extend the durability of its products.

-Increase the service intensity of its goods and services.

\section{CONCLUSIONS}

The development and optimization of many aspects of biofuel production technology can benefit from the work already accomplished through synthetic biology. One of the current challenges facing synthetic biology is the reproducibility of the developed tools in different systems. However, current technology developed by synthetic biology has already allowed for the successful design of many heterologously expressed biofuel production and substrate utilization pathways extending beyond the most user-friendly organisms.

The advancement of synthetic biology toward new diagnostic tools and high throughput screening systems will aid in the further development of these biofuel processes for pathway optimization and enzyme discovery or improvement. The success that synthetic biology has already afforded biofuel production technology lends confidence to future synergistic developments and breakthroughs.

The desire for renewable liquid fuel replacements to petroleum has steadily increased with concerns about the current fuel economy's stability and environmental impact. The development of new biofuel production processes has sought to mitigate some of these issues. The integration of synthetic biology with the development of these processes will be significant in bringing the biofuels industry from its infancy to a commercially viable alternative to petroleum.

\section{ACKNOWLEDGEMENTS}

This work was partly supported by the National Science and Technology Support Program of China under Grant No.2012BAC20B09. 


\section{REFERENCES}

[1] Savage D.F., Way J., Silver P.A. Defossiling fuel: how synthetic biology can transform biofuel production. ACS Chem. Biol., 2008; 3: 13-16.

[2] Purnick P.E.M., Weiss R. The second wave of synthetic biology: from module to systems. Nat. Rev. Mol. Cell Biol., 2009; 10: 410-422.

[3] Schmidt C.W. Synthetic biology: environmental health implications of a new field. Environ Health Perspect, 2010; 118: A118-A123.

[4] Kale, D.K., Anthappan, P.D. Sustainable Treatment of Wastewater using Effective Micro- organisms. Journal of Pure and Applied Micro-biology, 2012; 6 (1): 333-36.

[5] Babu, R.S., Prasad, S.B.C., Chakrapani, R., Padmavathi, M., Rao, C.S.V.R, Narasu, M.L. Studies on Bioethanol Production from Spoiled Starch Rich and Cellulose Rich Vegetables by Two Stage Batch Fermentation. Journal of Pure and Applied Microbiology, 2011; 5 (2): 853-57.

[6] David Houle, Diddahally R. Govindaraju and Stig Omholt. Phenomics: The Next Challenge, Nature Reviews Genetics, 2010;11: 855-866.

[7] Philippe Marguet, Frederick Balagadde, Cheemeng Tan, and Lingchong You. Biology by Design: Reduction and Synthesis of Cellular Components and Behaviour. Journal of The Royal Society Interface, 2007; 4: 607-623.

[8] Bong Hyun Sung, Jun Hyoung Lee and Sun Chang Kim. Escherichia coli Genome Engineering and Minimization for the Construction of a Bioengine. In: Systems Biology and Biotechnology of Escherichia coli, Springer Netherlands, 19-40, 2009.

[9] Mohammed S. El-Hersh, Husain A. El-Fadaly, Wesam I.A. Saber and Amany M. El-Deeb. Human Diseases Prosecution among Viral Infection and Food Toxins: A Review. International Journal of Pharmacology, 2013; 9(7): 390-404.

[10] Soodabeh Saeidnia and Mohammad Abdollahi. Perspective Studies on Novel Anticancer Drugs from Natural Origin: A Comprehensive Review. International Journal of Pharmacology, 2014; 10(2): 90-108.

[11] Secretary-General of the OECD, "Synthesis Report", Symposium on Opportunities and Challenges in the
Emerging Field of Synthetic Biology, OECD \& the Royal Society, 2010.

[12] Thomas H. Murray, Society Must Weigh "Massive" Potential Benefits Against Risks of Synthetic Life. See http://www.aaas.org/news/ releases/2010/1116hitachi_synth_bio.shtml

[13] See http://www.easac.eu, the European Academies Science Advisory Council, January 2011.

[14] Kwok, R., Five Hard Truths for Synthetic Biology, Nature, 2010; 463(7279): 288-290.

[15] Ying Jian Chen. Role of Liquid Biofuels in Realizing of Global Sustainability. Asian Journal of Chemistry, 2014; 26(11): 3355-3360.

[16] Fortman, J.L., Swapnil Chhabra, Aindrila Mukhopadhyay, Howard Chou, Taek Soon Lee, Eric Steen and Jay D. Keasling, Biofuel Alternatives to Ethanol: Pumping the Microbial Well. Trends Biotechnol, 2008; 26: 375-381.

[17] G. Ramya, R. Vaithiyanathan, J. Amala Infant Joice, R. Sudhakar and T. Sivakumar, Application of Core-Shell Modeled Composite Catalysts for Biofuel Production from Non-edible Vegetable Oils. Asian Journal of Chemistry, 2012; 24(12): 5683-5686.

[18] Alper, H., Stephanopoulos, G., Engineering for Biofuels: Exploiting Innate Microbial Capacity or Importing Biosynthetic Potential? Nature Rev. Microbiol., 2009; 7: 715-723.

[19] Lee, S. K., Chou, H., Ham, T. S., Lee, T. S., Keasling, J. D., Metabolic Engineering of Micro-organisms for Biofuels Production: From Bugs to Synthetic Biology to Fuels. Current Opinion in Biotechnology, 2008; 19: 556563.

[20] Guido, N.J., Wang, X., Adalsteinsson, D., et al., A bottom-up approach to gene regulation. Nature, 2006; 439(7078): 856-860.

[21] Gibson, D.G., Glass, J.I., Lartigue, C., et al., Creation of a bacterial cell controlled by a chemically synthesized genome. Science, 2010; 329(5987): 52-56.

[22] Livio D. DeSimone and Frank Popoff, Eco-efficiency: The Business Link to Sustainable Development, MIT Press, 1997; pp1-23. 